\title{
Energy and angular momentum of the gravitational field in the teleparallel geometry
}

\author{
J. W. Maluf, ${ }^{*}$ J. F. da Rocha-Neto, ${ }^{\dagger}$ T. M. L. Toríbio, and K. H. Castello-Branco \\ Instituto de Física, Universidade de Brasília, C.P. 04385, 70.919-970 Brasília DF, Brazil
}

(Received 1 October 2001; published 17 May 2002)

\begin{abstract}
The Hamiltonian formulation of the teleparallel equivalent of general relativity is considered. Definitions of energy, momentum and angular momentum of the gravitational field arise from the integral form of the constraint equations of the theory. In particular, the gravitational energy-momentum is given by the integral of scalar densities over a three-dimensional spacelike hypersurface. The definition for the gravitational energy is investigated in the context of the Kerr black hole. In the evaluation of the energy contained within the external event horizon of the Kerr black hole, we obtain a value strikingly close to the irreducible mass of the latter. The gravitational angular momentum is evaluated for the gravitational field of a thin, slowly rotating mass shell.

DOI: 10.1103/PhysRevD.65.124001

PACS number(s): 04.20.Cv, 04.20.Fy, 04.90.+e
\end{abstract}

\section{INTRODUCTION}

Teleparallel theories of gravity have been considered a long time ago in connection with attempts to define the energy of the gravitational field [1]. By studying the properties of solutions of Einstein's equations that describe the gravitational field of isolated material systems, it is concluded that a consistent expression for the energy density of the gravitational field would be given in terms of second order derivatives of the metric tensor. It is known that there exists no covariant, nontrivial expression constructed out of the metric tensor, both in three and in four dimensions, that contains such derivatives. However, covariant expressions that contain second order derivatives of tetrad fields are feasible. Thus it is legitimate to conjecture that the difficulties regarding the problem of defining the gravitational energymomentum is related to the geometrical description of the gravitational field, rather than being an intrinsic drawback of the theory [2].

It is usually asserted in the literature that the principle of equivalence prevents the localizability of the gravitational energy. However, an expression for the gravitational field energy has been pursued since the early days of general relativity. A considerable amount of effort has been devoted to finding viable expressions other than pseudotensors (more recently the idea of quasilocal energy, i.e., energy associated to a closed spacelike two-surface, in the context of the Hilbert-Einstein action integral, has emerged as a tentative description of the gravitational energy [3]). The search for a consistent expression for the gravitational energy is undoubtedly a long-standing problem in general relativity. The argument based on the principle of equivalence regarding the nonlocalizability of the gravitational energy is controversial and not generally accepted [2]. The principle of equivalence does not preclude the existence of scalar densities on the space-time manifold, constructed out of tetrad (or triad) fields, that may eventually yield the correct description of the

\footnotetext{
*Email address: wadih@ fis.unb.br

${ }^{\dagger}$ Present address: Instituto de Física Teórica, Universidade Estadual Paulista, Rua Pamplona 145, 01405-900 São Paulo, SP, Brazil.
}

energy properties of the gravitational field. Such densities may be given in terms of the torsion tensor, which cannot be made to vanish at a point by a coordinate transformation. Møller [1] was probably the first one to notice that the tetrad description of the gravitational field allows a more satisfactory treatment of the gravitational energy-momentum.

The dynamics of the gravitational field can be described in the context of the teleparallel geometry, where the basic geometrical entity is the tetrad field $e^{a}{ }_{\mu}[a$ and $\mu$ are $\mathrm{SO}(3,1)$ and space-time indices, respectively]. Teleparallel theories of gravity are defined on the Weitzenböck spacetime [4], endowed with the affine connection

$$
\Gamma_{\mu \nu}^{\lambda}=e^{a \lambda} \partial_{\mu} e_{a \nu}
$$

The curvature tensor constructed out of Eq. (1.1) vanishes identically. This connection defines a space-time with teleparallelism, or absolute parallelism [5]. This geometrical framework was considered by Einstein [6] in his attempt at unifying gravity and electromagnetism.

Gravity theories in this geometrical framework are constructed out of the torsion tensor. An infinity of such theories defined by a Lagrangian density, quadratic in the torsion tensor, has been investigated by Hayashi and Shirafuji [7] (who denote $e^{a}{ }_{\mu}$ as parallel vector fields). Among such infinity of theories a particular one is distinguished, because the tetrad fields that are solutions of this particular theory yield a metric tensor that is a solution of Einstein's equations. The teleparallel equivalent of general relativity (TEGR) [8-14] constitutes an alternative geometrical description of Einstein's equations.

A simple expression for the gravitational energy arises in the Hamiltonian formulation of the TEGR [13] in the framework of Schwinger's time gauge condition [15]. The energy density is given by a scalar density in the form of a total divergence that appears in the Hamiltonian constraint of the theory [16]. The investigations carried out so far confirm the consistency and relevance of this energy expression.

A recent approach to the localization of the gravitational energy has been considered in the Lagrangian framework of the TEGR by Andrade, Guillen and Pereira [17]. The existence of an expression for the gravitational energy density 
that is a true space-time tensor, and that reduces to Møller's energy-momentum density of the gravitational field was shown in [17].

The Hamiltonian formulation of the TEGR, with no $a$ priori restriction on the tetrad fields, has recently been established [18]. Its canonical structure is different from that obtained in Ref. [13], since it is not given in the standard Arnowitt-Deser-Misner (ADM) form [19]. In this framework we again arrive at an expression for the gravitational energy, in strict similarity with the procedure adopted in Ref. [16], namely, by interpreting the Hamiltonian constraint equation as an energy equation for the gravitational field. Likewise, the gravitational momentum can be defined. The constraint algebra of the theory suggests that certain momentum components are related to the gravitational angular momentum. It turns out to be possible to define, in this context, the angular momentum of the gravitational field.

In this article we investigate the definition of gravitational energy that arises in Ref. [18], in the framework of the Kerr metric tensor [20]. The whole formulation developed in Ref. [18] is carried out without enforcing the time gauge condition. It turns out, however, that consistent values for the gravitational energy are achieved by requiring the tetrad field to satisfy (a posteriori) the time gauge condition.

We investigate the irreducible mass $M_{i r r}$ of the Kerr black hole. It is the total mass of the black hole at the final stage of Penrose's process of energy extraction, considering that the maximum possible energy is extracted. It is also related to the energy contained within the external event horizon $E\left(r_{+}\right)$of the black hole (the surface of constant radius $r$ $=r_{+}$defines the external event horizon). Every expression for local or quasilocal gravitational energy must necessarily yield the value of $E\left(r_{+}\right)$in close agreement with $2 M_{i r r}$, since we know beforehand the value of the latter as a function of the initial angular momentum of the black hole [21]. The evaluation of $2 M_{i r r}$ is a crucial test for any expression for the gravitational energy. $E\left(r_{+}\right)$has been obtained by means of different energy expressions in Ref. [22]. Our expression for the gravitational energy is the only one that yields a satisfactory value for $E\left(r_{+}\right)$, strikingly close to $2 M_{i r r}$, and that arises in the framework of the Hamiltonian formulation of the gravitational field.

In the Hamiltonian formulation of the TEGR [18] there arises a set of primary constraints $\Gamma^{i k}$ that satisty the angular momentum algebra. Following the prescription for defining the gravitational energy, the definition of the gravitational angular momentum arises by suitably interpreting the integral form of the constraint equation $\Gamma^{i k}=0$ as an angular momentum equation. We apply this definition to the gravitational field of a thin, slowly rotating mass shell. In the limit of slow rotation we obtain a realistic measure of the angular momentum of the field in terms of the moment of inertia of the source.

Notation: space-time indices $\mu, \nu, \ldots$ and $\mathrm{SO}(3,1)$ indices $a, b, \ldots$ run from 0 to 3 . Time and space indices are indicated according to $\mu=0, i, \quad a=(0),(i)$. The tetrad field $e^{a}{ }_{\mu}$ yields the definition of the torsion tensor: $T_{\mu \nu}^{a}=\partial_{\mu} e^{a}{ }_{\nu}$ $-\partial_{\nu} e_{\mu}^{a}$. The flat, Minkowski space-time metric is fixed by $\eta_{a b}=e_{a \mu} e_{b \nu} g^{\mu \nu}=(-+++)$.

\section{THE HAMILTONIAN CONSTRAINT EQUATION AS AN ENERGY EQUATION FOR THE GRAVITATIONAL FIELD}

We summarize here the Hamiltonian formulation obtained in Ref. [13], where Schwinger's time gauge is assumed. The Hamiltonian density constructed out of triads $e_{(i) j}$ restricted to the three-dimensional spacelike hypersurface, and of the momenta canonically conjugated $\Pi^{(i) j}$, is given by

$$
\begin{aligned}
H= & N C+N^{i} C_{i}+\Sigma_{m n} \Pi^{m n} \\
& +\frac{1}{8 \pi G} \partial_{k}\left(N e T^{k}\right)+\partial_{k}\left(\Pi^{j k} N_{j}\right),
\end{aligned}
$$

where $N$ and $N^{i}$ are lapse and shift functions, $\Sigma_{m n}=-\Sigma_{n m}$ are Lagrange multipliers, $G$ is the gravitational constant and $\Pi^{i j}=e_{(k)}{ }^{i} \Pi^{(k) j}$. The constraints are defined by

$$
\begin{aligned}
C= & \partial_{j}\left(2 k e T^{j}\right)-k e \Sigma^{k i j} T_{k i j} \\
& -\frac{1}{4 k e}\left(\Pi^{i j} \Pi_{j i}-\frac{1}{2} \Pi^{2}\right), \\
C_{k}= & -e_{(j) k} \partial_{i} \Pi^{(j) i}-\Pi^{(j) i} T_{(j) i k},
\end{aligned}
$$

where $e=\operatorname{det}\left(e_{(i) j}\right)$ and $k=1 / 16 \pi G$. The tensor $\Sigma^{k i j}$ reads

$$
\Sigma^{k i j}=\frac{1}{4}\left(T^{k i j}+T^{i k j}-T^{j k i}\right)+\frac{1}{2}\left(g^{k j} T^{i}-g^{k i} T^{j}\right) .
$$

The trace of the torsion tensor is $T^{i}=g^{i k} T_{k}$ $=g^{i k} e^{(m) j} T_{(m) j k}$. The definition of $\sum^{k i j}$ yields

$$
\Sigma^{k i j} T_{k i j}=\frac{1}{4} T^{k i j} T_{k i j}+\frac{1}{2} T^{k i j} T_{i k j}-T^{i} T_{i} .
$$

The first two terms on the right-hand side of Eq. (2.2) are equivalent to the scalar curvature $R\left(e_{(i) j}\right)$ on the threedimensional spacelike hypersurface,

$$
2 \partial_{j}\left(e T^{j}\right)-e \Sigma^{k i j} T_{k i j}=e R\left(e_{(i) j}\right) .
$$

The integral form of the Hamiltonian constraint equation $C(x)=0$ can be interpreted as an energy equation [16],

$$
\begin{aligned}
\int d^{3} x \partial_{j}\left(2 k e T^{j}\right)= & \int d^{3} x\left\{k e \Sigma^{k i j} T_{k i j}\right. \\
& \left.+\frac{1}{4 k e}\left(\Pi^{i j} \Pi_{j i}-\frac{1}{2} \Pi^{2}\right)\right\} .
\end{aligned}
$$

We identify Eq. (2.6) as an energy equation because the integral of the left-hand side of this equation over the whole three-dimensional space yields the Arnowitt-Deser-Misner energy [19],

$$
\begin{aligned}
\frac{1}{8 \pi G} \int d^{3} x \partial_{j}\left(e T^{j}\right) & =\frac{1}{16 \pi G} \int_{S} d S_{k}\left(\partial_{i} h_{i k}-\partial_{k} h_{i i}\right) \\
& =E_{A D M} .
\end{aligned}
$$


The right-hand side of Eq. (2.7) is obtained by requiring the asymptotic behavior

$$
e_{(i) j} \simeq \eta_{i j}+\frac{1}{2} h_{i j}\left(\frac{1}{r}\right),
$$

in the limit $r \rightarrow \infty . \eta_{i j}$ is the spatial sector of Minkowski's metric tensor and $h_{i j}$ is the first term in the asymptotic expansion of $g_{i j}$. Therefore we define the gravitational energy enclosed by a volume $V$ of the three-dimensional space as [16]

$$
E_{g}=\frac{1}{8 \pi G} \int_{V} d^{3} x \partial_{j}\left(e T^{j}\right)
$$

The expression above has been applied to several configurations of the gravitational field. The most relevant application is the evaluation of the irreducible mass of the Kerr black hole [23].

\section{GRAVITATIONAL ENERGY EXPRESSION IN TERMS OF TETRAD FIELDS}

An expression for the gravitational energy density also arises in the framework of the Hamiltonian formulation of general relativity in the teleparallel geometry [18], without posing any a priori restriction on the tetrad fields, again interpreting the integral form of the constraint equations as energy-momentum equations for the gravitational field.

The Hamiltonian formulation developed in Ref. [18] is obtained from the Lagrangian density in empty space-time defined by

$$
L(e)=-k e\left(\frac{1}{4} T^{a b c} T_{a b c}+\frac{1}{2} T^{a b c} T_{b a c}-T^{a} T_{a}\right),
$$

where $e=\operatorname{det}\left(e^{a}{ }_{\mu}\right), \quad T_{a b c}=e_{b}{ }^{\mu} e_{c}{ }^{\nu} T_{a \mu \nu}$, and the trace of the torsion tensor is given by $T_{b}=T_{a b}^{a}$. The Hamiltonian is obtained by just rewriting the Lagrangian density in the form $L=p \dot{q}-H$. It has not been made use of any kind of projection of metric variables to the three-dimensional spacelike hypersurface. Since there is no time derivative of $e_{a 0}$ in Eq. (3.1), the corresponding momentum canonically conjugated $\Pi^{a 0}$ vanishes identically. Dispensing with surface terms the total Hamiltonian density reads [18]

$$
H\left(e_{a i}, \Pi^{a i}\right)=e_{a 0} C^{a}+\alpha_{i k} \Gamma^{i k}+\beta_{k} \Gamma^{k},
$$

where $\left\{C^{a}, \Gamma^{i k}\right.$ and $\left.\Gamma^{k}\right\}$ constitute a set of primary constraints, and $\alpha_{i k}$ and $\beta_{k}$ are Lagrange multipliers. Explicit details are given in Ref. [18]. The first term of the constraint $C^{a}$ is given by a total divergence in the form $C^{a}=-\partial_{k} \Pi^{a k}$ $+\cdots$. In similarity with Eq. (2.6) we identify this total divergence on the three-dimensional spacelike hypersurface as the energy-momentum density of the gravitational field. The total energy-momentum is defined by

$$
P^{a}=-\int_{V} d^{3} x \partial_{i} \Pi^{a i}
$$

where $V$ is an arbitrary space volume. It is invariant under coordinate transformations on the spacelike manifold, and transforms as a vector under the global $\mathrm{SO}(3,1)$ group (we will return to this point later on). The definition above generalizes expression (2.9) to tetrad fields that are not restricted by the time gauge condition. However, both expressions are equivalent, as we will see ahead, if the time gauge condition is imposed. After implementing the primary constraints $\Gamma^{i k}$ and $\Gamma^{k}$, the expression of the momenta $\Pi^{a k}$ reads

$$
\begin{aligned}
\Pi^{a k}= & k e\left\{g^{00}\left(-g^{k j} T^{a}{ }_{0 j}-e^{a j} T^{k}{ }_{0 j}+2 e^{a k} T^{j}{ }_{0 j}\right)+g^{0 k}\left(g^{0 j} T^{a}{ }_{0 j}\right.\right. \\
& \left.+e^{a j} T_{0 j}^{0}\right)+e^{a 0}\left(g^{0 j} T^{k}{ }_{0 j}+g^{k j} T^{0}{ }_{0 j}\right)-2\left(e^{a 0} g^{0 k} T^{j}{ }_{0 j}\right. \\
& \left.+e^{a k} g^{0 j} T^{0}{ }_{0 j}\right)-g^{0 i} g^{k j} T^{a}{ }_{i j}+e^{a i}\left(g^{0 j} T^{k}{ }_{i j}-g^{k j} T^{0}{ }_{i j}\right) \\
& \left.-2\left(g^{0 i} e^{a k}-g^{i k} e^{a 0}\right) T^{j}{ }_{j i}\right\} .
\end{aligned}
$$

With appropriate boundary conditions, expression (3.3) yields the ADM energy. Let us consider asymptotically flat space-times and assume that in the limit $r \rightarrow \infty$ the tetrad fields have the asymptotic behavior

$$
e_{a \mu} \simeq \eta_{a \mu}+\frac{1}{2} h_{a \mu}\left(\frac{1}{r}\right)
$$

where $\eta_{a \mu}$ is Minkowski's metric tensor and $h_{a \mu}$ is the first term in the asymptotic expansion of $g_{\mu \nu}$. Asymptotically flat space-times are defined by Eq. (3.5) together with $\partial_{\mu} g_{\lambda \nu}$ $=O\left(1 / r^{2}\right)$, or $\partial_{\mu} e_{a \nu}=O\left(1 / r^{2}\right)$. Considering the $a=(0)$ component in Eq. (3.3) and integrating over the whole threedimensional spacelike hypersurface, we find, after a long but straightforward calculation, that

$$
\begin{aligned}
P^{(0)} & =E=-\int_{V \rightarrow \infty} d^{3} x \partial_{k} \Pi^{(0) k} \\
& =-2 k \int_{V \rightarrow \infty} d^{3} x \partial_{k}\left(e g^{i k} e^{(0) 0} T_{j i}^{j}\right) \\
& =\frac{1}{16 \pi G} \int_{S \rightarrow \infty} d S_{k}\left(\partial_{i} h_{i k}-\partial_{k} h_{i i}\right)=E_{A D M} .
\end{aligned}
$$

We will prove that expressions (2.9) and (3.3) coincide if we require the time gauge condition. In order to prove it, let us rewrite $\Pi^{(0) k}$ as

$$
\Pi^{(0) k}=e^{(0)}{ }_{i} \Pi^{(i k)}+e^{(0)}{ }_{i} \Pi^{[i k]}+e^{(0)}{ }_{0} \Pi^{0 k},
$$

where $(\cdots)$ and $[\cdots]$ denote symmetric and antisymmetric components, respectively. In the time gauge condition we have $e_{(j)}{ }^{0}=e^{(0)}{ }_{i}=0$, and therefore Eq. (3.7) reduces to $\Pi^{(0) k}=e^{(0)}{ }_{0} \Pi^{0 k}$. An expression for $\Pi^{0 k}$ can be obtained by requiring the vanishing of the constraint $\Gamma^{k}[18]$,

$$
\begin{aligned}
\Gamma^{k}= & \Pi^{0 k}+2 k e\left(g^{k j} g^{0 i} T^{0}{ }_{i j}-g^{0 k} g^{0 i} T^{j}{ }_{i j}\right. \\
& \left.+g^{00} g^{i k} T^{j}{ }_{i j}\right) .
\end{aligned}
$$

In the time gauge we have $T^{0}{ }_{i j}=0$ and therefore from $\Gamma^{k}$ $=0$ we arrive at 


$$
\Pi^{0 k}=2 k e\left(g^{0 k} g^{0 i}-g^{00} g^{i k}\right) T^{j}{ }_{i j} .
$$

All quantities in Eq. (3.9) are four-dimensional field quantities. Let us now rewrite Eq. (3.9) in terms of field quantities restricted to the three-dimensional spacelike hypersurface by means of the lapse and shift functions, $N$ and $N^{i}$, respectively. In view of the relations $e=N\left({ }^{3} e\right), g^{0 i}=N^{i} / N^{2}$, and $g^{i k}={ }^{3} g^{i k}-\left(N^{i} N^{k}\right) / N^{2}$, Eq. (3.9) can be written as

$$
\Pi^{0 k}=\frac{2}{N} k\left({ }^{3} e\right)\left({ }^{3} g^{i k}\right) T^{j}{ }_{i j} .
$$

The superscript 3 indicates that the quantity is projected on the spacelike hypersurface. Note that $T^{j}{ }_{i j}$ is still given in terms of four-dimensional field quantities.

We make use of a $3+1$ decomposition for the tetrad fields according to $e^{a i}={ }^{3} e^{a i}+\left(N^{i} / N\right) \eta^{a}, e^{a}{ }_{i}={ }^{3} e^{a}{ }_{i}, \eta^{a}=-N e^{a 0}$, and $e^{a}{ }_{0}=\eta^{a} N+{ }^{3} e^{a}{ }_{i} N^{i}$. The tetrad fields ${ }^{3} e_{a i}$ and ${ }^{3} e^{a i}$ are related to each other by means of the metric tensor $g_{i j}$ and its inverse ${ }^{3} g^{i j}$. With the help of these relations we can rewrite $T^{j}{ }_{i j}$ in terms of quantities on the spacelike hypersurface in the time gauge condition, in which case we have $\eta^{a}=\delta_{(0)}^{a}$ and $e^{(0)}{ }_{0}=N$. We eventually arrive at

$$
\Pi^{(0) k}=2 \operatorname{keg}^{i k} g^{j m} e^{(l)}{ }_{m} T_{(l) i j},
$$

where we have eliminated the superscript 3 . It is straightforward to verify that $\Pi^{(0) k}=-2 k T^{k}$, where $T^{k}$ and $T_{(l) i j}$ are precisely the same quantities that appear in Sec. II, and in particular in expression (2.9). Therefore in the time gauge condition we have

$$
P^{(0)}=-\int_{V} d^{3} x \partial_{i} \Pi^{(0) i}=\frac{1}{8 \pi G} \int_{V} d^{3} x \partial_{j}\left(e T^{j}\right) .
$$

Differently from the quasilocal energy expressions [3], Eq. (3.11) is an integral of a scalar density over finite space volumes, which can be transformed into a surface integral. Therefore our expression is not bound, in principle, to belong to any class of quasilocal energies. There is no need of subtraction terms in the present framework. And yet Eq. (3.11) does satisfy the usual requirements for a quasilocal energy expression. According to the latter requirements the quasilocal energy expression must (i) vanish for the Minkowski space-time; (ii) yield the ADM and Bondi mass in the appropriate limits; (iii) yield the appropriate value for weak and spherically symmetric gravitational fields; and (iv) yield the irreducible mass of the Kerr black hole. The Bondi energy in the TEGR has been discussed in Ref. [24], and the latter requirement is discussed in Sec. V.

\section{THE DETERMINATION OF TETRAD FIELDS}

In the framework of the teleparallel geometry, the gravitational field can be described by an anholonomic transformation between a reference space-time and the physical space-time. We will briefly recall the difference between holonomic and anholonomic transformations. Let us consider two sets of coordinates, $q^{a}=(t, x, y, z)$ and $x^{\mu}=(t, r, \theta, \phi)$, related by the coordinate transformation $d q^{a}=e^{a}{ }_{\mu} d x^{\mu}$ such that

$$
\begin{aligned}
e_{\mu}^{a} & =\frac{\partial q^{a}}{\partial x^{\mu}} \\
& =\left(\begin{array}{cccc}
1 & 0 & 0 & 0 \\
0 & \sin \theta \cos \phi & r \cos \theta \cos \phi & -r \sin \theta \sin \phi \\
0 & \sin \theta \sin \phi & r \cos \theta \sin \phi & r \sin \theta \cos \phi \\
0 & \cos \theta & -r \sin \theta & 0
\end{array}\right) .
\end{aligned}
$$

The relation $d q^{a}=e^{a}{ }_{\mu} d x^{\mu}$ can be integrated over the whole space-time, and therefore the transformation $q^{a} \rightarrow x^{\mu}$ corresponds to a single-valued global transformation. In this case the transformation is called holonomic and both coordinate sets describe the same space-time.

However, in the general case the relation $d q^{a}=e^{a}{ }_{\mu} d x^{\mu}$ cannot be globally integrated, since $e^{a}{ }_{\mu}$ may not be a gradient function of the type $\partial_{\mu} q^{a}$. If the quantities $e_{\mu}^{a}$ are such that $\partial_{\mu} e^{a}{ }_{\nu}-\partial_{\nu} e_{\mu}^{a} \neq 0$, then the transformation is called anholonomic.

For the tetrads given by Eq. (4.1) the torsion tensor $T^{a}{ }_{\mu \nu}=\partial_{\mu} e^{a}{ }_{\nu}-\partial_{\nu} e^{a}{ }_{\mu}$ vanishes. It is known that $T^{a}{ }_{\mu \nu}$ vanish identically if and only if $e^{a}$ are gradient vectors [25]. In the framework of the TEGR the gravitational field corresponds to a configuration such that $T_{\mu \nu}^{a} \neq 0$. Thus every gravitational field is described by a space-time that is anholonomically related to the four-dimensional Minkowski space-time, which is taken as the reference space-time. Consequently the tetrad fields to be considered must necessarily yield a vanishing torsion tensor in the limit of vanishing physical parameters (such as mass, angular momentum and charge), in which case the tetrad field must reduce to expression (4.1), or, in the case of arbitrary coordinates, to the form $e^{a}{ }_{\mu}$ $=\partial_{\mu} q^{a}$.

The idea of describing the gravitational field as the gauge field of the Poincaré group is rather widespread. In view of the general acceptance of this idea, there is an unjustified prejudice against gravitational theories that do not exhibit local SO(3,1) symmetry. Rather than being a drawback of the present formulation, the requirement of a global set of tetrad fields for the description of the space-time is a natural feature of teleparallel theories [6] and of the teleparallel geometry.

Before addressing the problem of obtaining the appropriate set of tetrad fields out of a given metric tensor, it is instructive to analyze the construction of tetrads for the flat space-time, since a number of features that take place in this context carry over to the general case of an arbitrary spacetime metric tensor. We will consider two sets of tetrads that describe the flat space-time, and that reveal the relationship between the reference space-time with coordinates $q^{a}$ and the physical space-time with coordinates $x^{\mu}$.

For a given arbitrary function $\omega(t)$ let us consider a transformation between two rotating Cartesian coordinate sys- 
tems, $\quad q^{0}=t, \quad q^{1}=x^{1} \cos \omega(t)-x^{2} \sin \omega(t), \quad q^{2}=x^{1} \sin \omega(t)$ $+x^{2} \cos \omega(t), \quad q^{3}=x^{3}$. The tetrads are given by

$$
\begin{aligned}
& e_{\mu}^{a}(t, x, y, z) \\
& =\left(\begin{array}{cccc}
1 & 0 & 0 & 0 \\
-\left(x^{1} \sin \omega+x^{2} \cos \omega\right) \dot{\omega} & \cos \omega & -\sin \omega & 0 \\
\left(x^{1} \cos \omega-x^{2} \sin \omega\right) \dot{\omega} & \sin \omega & \cos \omega & 0 \\
0 & 0 & 0 & 1
\end{array}\right) .
\end{aligned}
$$

These tetrads describe a flat space-time with Cartesian coordinates $x^{\mu}$ that is rotating with respect to the reference spacetime with coordinates $q^{a}$. We notice the appearance of antisymmetric components in the spatial sector of $e^{a}{ }_{\mu}$. This is a general feature in Cartesian coordinates: under an infinitesimal rotation, a rotated vector $\widetilde{V}$ is related to the vector $V$ by means of the relation $\widetilde{V}=R V$; the rotation matrix is given by $R=1+\omega_{i} X^{i}$, where $\omega_{i}$ are arbitrary parameters and the generators $X^{i}$ are anti-symmetric matrices. Therefore the emergence of anti-symmetric components in the sector $e_{(i) j}(t, x, y, z)$ is expected if the two space-times are rotating with respect to each other.

Another transformation of general character is a Lorentz boost, $\quad q^{(0)}=\gamma\left[t+\left(v / c^{2}\right) x^{1}\right], \quad q^{(1)}=\gamma\left(x^{1}+v t\right), \quad q^{(2)}=x^{2}$, and $q^{(3)}=x^{3}$, where $\gamma=1 / \sqrt{1-v^{2} / c^{2}}$ (assuming the velocity of light $c \neq 1$ ). The two space-times have different time scales. The tetrads read

$$
e_{\mu}^{a}(t, x, y, z)=\left(\begin{array}{cccc}
\gamma & \left(v / c^{2}\right) \gamma & 0 & 0 \\
v \gamma & \gamma & 0 & 0 \\
0 & 0 & 1 & 0 \\
0 & 0 & 0 & 1
\end{array}\right) .
$$

The tetrads above do not satisfy the time gauge condition because of the emergence of the term $e^{(0)}{ }_{1}=\left(v / c^{2}\right) \gamma$. Under an arbitrary boost transformation there will arise terms such that $e^{(0)}{ }_{k} \neq 0$, which violate the time gauge condition $e_{(i)}{ }^{0}$ $=0$. The main feature of the time gauge condition is to lock the time axes of the reference space-time and of the physical space-time.

In the absence of the gravitational field, $e^{a}{ }_{\mu}(t, x, y, z)$ $=\delta_{\mu}^{a}$ is the unique set of tetrads that describes a reference space-time with coordinates $q^{a}$ that is neither related by a boost transformation nor rotating with respect to the physical space-time with coordinates $x^{\mu}$. The features above should also carry over to the case of an arbitrary gravitational field. As we will see, they are essential in the description of the energy properties of the gravitational field. Likewise, for a given space-time metric tensor the set of tetrad fields that in Cartesian coordinates satisfy the properties

$$
e_{(i) j}=e_{(j) i},
$$

$$
e_{(i)}{ }^{0}=0
$$

establish a unique reference space-time that is neither related by a boost transformation, nor rotating with respect to the physical space-time. Equation (4.4b) fixes six degrees of freedom of the tetrad field. The importance of Eqs. (4.4a),(4.4b) to the definition of the gravitational energy will be discussed at the end of Sec. V.

Let us consider now the Kerr space-time. In terms of Boyer-Lindquist [26] coordinates the Kerr metric tensor is given by

$$
\begin{aligned}
d s^{2}= & -\frac{\psi^{2}}{\rho^{2}} d t^{2}-\frac{2 \chi \sin ^{2} \theta}{\rho^{2}} d \phi d t+\frac{\rho^{2}}{\Delta} d r^{2} \\
& +\rho^{2} d \theta^{2}+\frac{\Sigma^{2} \sin ^{2} \theta}{\rho^{2}} d \phi^{2}
\end{aligned}
$$

where $\rho^{2}=r^{2}+a^{2} \cos ^{2} \theta, \quad \Delta=r^{2}+a^{2}-2 m r, \quad \chi=2 a m r$ and

$$
\begin{gathered}
\Sigma^{2}=\left(r^{2}+a^{2}\right)^{2}-\Delta a^{2} \sin ^{2} \theta, \\
\psi^{2}=\Delta-a^{2} \sin ^{2} \theta .
\end{gathered}
$$

Each set of tetrad fields defines a teleparallel geometry. For a given space-time metric tensor $g_{\mu \nu}$, there exists an infinite set of tetrad fields that yield $g_{\mu \nu}$. From the point of view of the metrical properties of the space-time, any two sets of tetrads out of this infinity correspond to viable (but distinct) teleparallel configurations [12]. However, the description of the gravitational field energy requires at least boundary conditions. In the framework of the teleparallel geometry the correct description of the gravitational energymomentum singles out a unique set of tetrad fields. In the following we will consider the most relevant tetrad configurations. The first one is based on the weak field approximation first suggested by Møller, given by expression (3.5),

$$
e_{a \mu}^{M} \simeq \eta_{a \mu}+\frac{1}{2} h_{a \mu},
$$

together with the symmetry condition on $h_{a \mu}$,

$$
h_{a \mu}=h_{\mu a} .
$$

Note that Eq. (4.6a) is demanded not only in the asymptotic limit, but at every space-time point. Although the weak field limit fixes the expression of $e_{a \mu}^{M}$, the resulting expression is taken to hold in the strong field regime. The expression that satisfies Eq. (4.6) and that yields Eq. (4.5) is given by 


$$
e_{a \mu}^{M}=\left(\begin{array}{cccc}
-\frac{\psi}{\rho} \sqrt{1+M^{2} y^{2}} & 0 & 0 & -\frac{\chi N y}{\psi \rho} \sin ^{2} \theta \\
\frac{\chi y}{\Sigma \rho} \sin \theta \sin \phi & \frac{\rho}{\sqrt{\Delta}} \sin \theta \cos \phi & \rho \cos \theta \cos \phi & -\frac{\Sigma}{\rho} \sqrt{1+M^{2} N^{2} y^{2}} \sin \theta \sin \phi \\
-\frac{\chi y}{\Sigma \rho} \sin \theta \cos \phi & \frac{\rho}{\sqrt{\Delta}} \sin \theta \sin \phi & \rho \cos \theta \sin \phi & \frac{\Sigma}{\rho} \sqrt{1+M^{2} N^{2} y^{2}} \sin \theta \cos \phi \\
0 & \frac{\rho}{\sqrt{\Delta}} \cos \theta & -\rho \sin \theta & 0
\end{array}\right),
$$

where

$$
\begin{gathered}
y^{2}=\frac{2 N \sqrt{1+M^{2}}-\left(1+N^{2}\right)}{4 M^{2} N^{2}-\left(1-N^{2}\right)^{2}}, \\
M=\frac{\chi}{\sum \psi} \sin \theta, \\
N=\frac{\psi r}{\Sigma} .
\end{gathered}
$$

$$
e_{(i) j} \simeq \eta_{i j}+\frac{1}{2} h_{i j}
$$

$$
h_{i j}=h_{j i},
$$

together with Schwinger's time gauge condition, $e_{(k)}{ }^{0}$ $=e^{(0)}=0$ [Eq. (4.4b)]. Note that Eqs. (4.8a), (4.8b) are essentially equivalent to Eq. (4.4a). Conditions (4.8) are assumed to fix the expression of $e^{a}{ }_{\mu}$ also in the strong field regime. The set of tetrad fields that satisfies Eqs. (4.8), (4.4b) and that yields Eq. (4.5) reads

$$
e_{a \mu}^{S}=\left(\begin{array}{cccc}
-\frac{1}{\rho} \sqrt{\psi^{2}+\frac{\chi^{2}}{\Sigma^{2}} \sin ^{2} \theta} & 0 & 0 & 0 \\
\frac{\chi}{\Sigma \rho} \sin \theta \sin \phi & \frac{\rho}{\sqrt{\Delta}} \sin \theta \cos \phi & \rho \cos \theta \cos \phi & -\frac{\Sigma}{\rho} \sin \theta \sin \phi \\
-\frac{\chi}{\Sigma \rho} \sin \theta \cos \phi & \frac{\rho}{\sqrt{\Delta}} \sin \theta \sin \phi & \rho \cos \theta \sin \phi & \frac{\Sigma}{\rho} \sin \theta \cos \phi \\
0 & \frac{\rho}{\sqrt{\Delta}} \cos \theta & -\rho \sin \theta & 0
\end{array}\right) .
$$

We note finally that both Eqs. (4.7) and (4.9) reduce to Eq. (4.1) if we make $m=a=0$.

\section{THE IRREDUCIBLE MASS OF THE KERR BLACK HOLE}

In this section we will apply expression (3.3) to the evaluation of the irreducible mass $M_{i r r}$ of the Kerr black hole. This is the most important test for any gravitational energy expression, local or quasilocal, since the geometrical setting corresponds to an intricate configuration of the gravitational field, and since the value of $M_{i r r}$ is known from the work of Christodoulou [21].

In order to obtain $M_{i r r}$ we will calculate the $a=(0)$ component of Eq. (3.3) by fixing $V$ to be the volume within the $r=r_{+}$surface, where $r_{+}=m+\sqrt{m^{2}-a^{2}}$ is the external horizon of the Kerr black hole. Therefore we will consider

$$
P^{(0)}=E=-\int_{S} d S_{i} \Pi^{(0) i}=-\int_{S} d \theta d \phi \Pi^{(0) 1}(r, \theta, \phi),
$$

where the surface $S$ is determined by the condition $r=r_{+}$. 


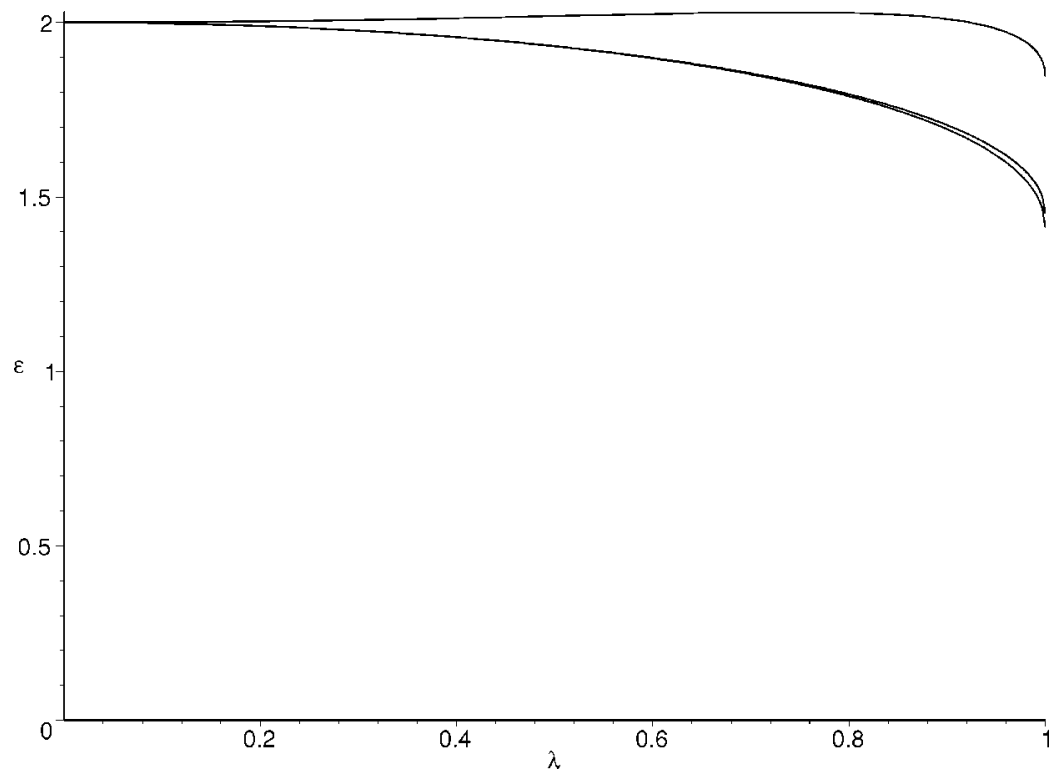

FIG. 1. Energy within the external event horizon of the Kerr black hole as a function of the angular momentum. The figure displays $\varepsilon=E / m$ against $\lambda$ for expressions (5.3) and (5.4). The lower curve represents $2 M_{i r r}$ given by Eq. (5.5). The one right above it, almost coinciding with the lower curve, corresponds to Eq. (5.4). The upper curve corresponds to Eq. (5.3).

The expression of $\Pi^{(0) 1}$ will be obtained by considering Eq. (4.7). In view of Eq. (3.11) there is no need to calculate Eq. (5.1) out of Eq. (4.9), since it has already been evaluated [23].

In the Appendix we present the expressions of the components of the torsion tensor constructed out of the tetrad configuration Eq. (4.7). The component $\Pi^{(0) 1}$ is then obtained from the definition (3.4) by means of simple (albeit long) algebraic manipulations. The expression of $\Pi^{(0) 1}(r, \theta, \phi)$ for the tetrad expression (4.7) reads

$$
\begin{aligned}
\Pi^{(0) 1}= & \frac{k \Sigma y}{\rho} \sin \theta\left\{-2\left(1+N \Omega+\frac{\rho^{2}}{y \Sigma}\right)+\frac{2 \sqrt{\Delta}}{\Sigma} \partial_{r} \Sigma\right. \\
& \left.+\frac{\sqrt{\Delta} N}{\Omega}\left(\frac{M^{2}}{\chi} \partial_{r} \chi+\frac{2}{\Sigma} \partial_{r} \Sigma\right)\right\},
\end{aligned}
$$

where the definitions of $y, N$, and $M$ are given after expression (4.7) and

$$
\Omega=\sqrt{1+M^{2}} .
$$

On the surface $r=r_{+}$we have $\Delta\left(r_{+}\right)=0, M^{2}\left(r_{+}\right)=-1$, and $\Omega\left(r_{+}\right)=0$. Therefore the last term in Eq. (5.2) is indefinite. It must be calculated by taking the limit $r \rightarrow r_{+}$. We find

$$
\begin{aligned}
\lim _{r \rightarrow r_{+}} & \frac{\sqrt{\Delta} N}{\Omega}\left(\frac{M^{2}}{\chi} \partial_{r} \chi+\frac{2}{\Sigma} \partial_{r} \Sigma\right) \\
& =-\frac{a^{2} \sin ^{2} \theta}{m}\left(\sqrt{\frac{m^{2}-a^{2}}{2 m r_{+}}}+\frac{r_{+}}{2 m r_{+}-a^{2} \sin ^{2} \theta}\right) .
\end{aligned}
$$

The other terms in Eq. (5.2) do not pose any problem, and thus we can obtain the expression of the energy contained within the external event horizon of the Kerr black hole, that follows from the tetrad configuration (4.7). The final expression arises as a function of the angular momentum per unit mass $a$. It is given by (we are assuming $G=1$ )

$$
\begin{aligned}
E\left[e_{a \mu}^{M}\right]= & \frac{m}{4} \int_{0}^{\pi} d \theta \sin \theta\left[\sqrt{p^{2}+\lambda^{2} \cos ^{2} \theta}\right. \\
& +\frac{p y}{\sqrt{p^{2}+\lambda^{2} \cos ^{2} \theta}}+\frac{2 p^{3} y}{\left(p^{2}+\lambda^{2} \cos ^{2} \theta\right)^{3 / 2}} \\
& \left.-\frac{y(p-1) \sqrt{p^{2}+\lambda^{2} \cos ^{2} \theta}}{2}\right],
\end{aligned}
$$

where

$$
p=1+\sqrt{1-\lambda^{2}}, \quad a=\lambda m, \quad 0 \leqslant \lambda \leqslant 1 .
$$

For the tetrad configuration Eq. (4.9) we have [23]

$$
E\left[e_{a \mu}^{S}\right]=m\left[\frac{\sqrt{2 p}}{4}+\frac{6 p-\lambda^{2}}{4 \lambda} \ln \left(\frac{\sqrt{2 p}+\lambda}{p}\right)\right] .
$$

Expressions (5.3) and (5.4) must be compared with $2 M_{i r r}$, where $M_{i r r}$ is given by [21] $M_{i r r}=\frac{1}{2} \sqrt{r_{+}^{2}+a^{2}}$. In our notation we have

$$
2 M_{i r r}=m \sqrt{2 p} \text {. }
$$

In the limit $a \rightarrow 0$ all energy expressions yield $2 m$, which is the value obtained by several different approaches [22]. In Fig. 1 we have plotted $\varepsilon=E / m$ against $\lambda$, where $0 \leqslant \lambda \leqslant 1$. Each value of $\lambda$ characterizes an angular momentum state of the black hole. The hope was that the tetrad field given by Eq. (4.7) would explain the tiny difference between the numerical values of Eqs. (5.4) and (5.5). However, the deviation of expression (5.3) from $2 M_{i r r}$ indicates that the tetrad configuration Eq. (4.7) is not appropriate to the description of gravitational energy. The latter is most correctly described by requiring the tetrad configuration Eq. (4.9), that satisfies Schwinger's time gauge condition together with Eq. (4.4a). 
The choice of the tetrad field given by Eq. (4.9) amounts to choosing the unique reference space-time that is neither related by a boost transformation nor rotating with respect to the physical space-time.

For an arbitrary space volume $V$ the gravitational energy is defined relationally, in the sense that it depends on the choice of the reference space-time. If the tetrad fields are required to satisfy conditions $(4.4 \mathrm{a}),(4.4 \mathrm{~b})$ for a metric tensor that exhibits asymptotic boundary conditions similar to Eq. (3.5), then for asymptotically flat space-times the physical space-time coincides with the reference space-time in the limit $r \rightarrow \infty$. If, however, we choose a reference space-time that is, for instance, rotating (about the $z$ axis, say) with respect to the space-time defined by the Kerr solution, then the irreducible mass of the black hole, calculated with respect to this reference space-time, will be different from expression (5.4), the difference residing in rotational effects. Therefore in similarity to the ordinary concept of energy, the gravitational energy depends on the rotational state of the reference frame. Rotational and boost effects are eliminted by requiring conditions $(4.4 \mathrm{a}),(4.4 \mathrm{~b})$ on the tetrad fields.

The agreement between Eqs. (5.4) and (5.5) is the most important result so far obtained from definitions (2.9) and (3.3). To our knowledge, the latter are the only energy definitions that yield a value satisfactorily close to $2 M_{i r r}$, and that arise from the structure of the Hamiltonian formulation of the theory.

Before closing this section we note that the time gauge condition $(4.4 b)$ breaks the $\mathrm{SO}(3,1)$ symmetry group into the global $\mathrm{SO}(3)$. Therefore in this case $P^{a}$ given by Eq. (3.3) is no longer a true $\mathrm{SO}(3,1)$ vector.

\section{ANGULAR MOMENTUM OF THE GRAVITATIONAL FIELD}

In the context of Einstein's general relativity, rotational phenomena is certainly not a completely understood issue. The prominent manifestation of a purely relativistic rotational effect is the dragging of inertial frames. If the angular momentum of the gravitational field of isolated systems has a meaningful notion, then it is reasonable to expect the latter to be somehow related to the rotational motion of the physical sources.

The angular momentum of the gravitational field has been addressed in the literature by means of different approaches. The oldest approach is based on pseudotensors [27,28], out of which angular momentum superpotentials are constructed. An alternative approach assumes the existence of certain Killing vector fields that allow the construction of conserved integral quantities [29]. Finally, the gravitational angular momentum can also be considered in the context of Poincaré gauge theories of gravity [30], either in the Lagrangian or in the Hamiltonian formulation. In the latter case it is required that the generators of spatial rotations at infinity have well defined functional derivatives. From this requirement a certain surface integral arises, whose value is interpreted as the gravitational angular momentum.

The main motivation for considering the angular momentum of the gravitational field in the present investigation re- sides in the fact that the constraints $\Gamma^{i k}[18]$,

$$
\begin{aligned}
\Gamma^{i k}= & -\Gamma^{k i} \\
= & 2 \Pi^{[i k]}-2 k e\left[-g^{i m} g^{k j} T^{0}{ }_{m j}\right. \\
& \left.+\left(g^{i m} g^{0 k}-g^{k m} g^{0 i}\right) T^{j}{ }_{m j}\right],
\end{aligned}
$$

satisfy the angular momentum algebra,

$$
\begin{aligned}
\left\{\Gamma^{i j}(x), \Gamma^{k l}(y)\right\}= & \left(g^{i l} \Gamma^{j k}+g^{j k} \Gamma^{i l}\right. \\
& \left.-g^{i k} \Gamma^{j l}-g^{j l} \Gamma^{i k}\right) \delta(x-y) .
\end{aligned}
$$

Following the prescription for defining the gravitational energy out of the Hamiltonian constraint of the TEGR, we interpret the integral form of the constraint equation $\Gamma^{i k}=0$ as an angular momentum equation, and therefore we define the angular momentum of the gravitational field $M^{i k}$ according to

$$
\begin{aligned}
M^{i k}= & 2 \int_{V} d^{3} x \Pi^{[i k]} \\
= & 2 k \int_{V} d^{3} x e\left[-g^{i m} g^{k j} T^{0}{ }_{m j}\right. \\
& \left.+\left(g^{i m} g^{0 k}-g^{k m} g^{0 i}\right) T^{j}{ }_{m j}\right],
\end{aligned}
$$

for an arbitrary volume $V$ of the three-dimensional space.

In Einstein-Cartan type theories there also appear constraints that satisfy the Poisson bracket given by Eq. (6.2). However, such constraints arise in the form $\Pi^{[i k]}=0$, and so a definition similar to Eq. (6.3), i.e., interpreting the constraint equation as an equation for the angular momentum of the field, is not possible.

Since definition (6.3) is a three-dimensional integral we will consider a non-singular space-time metric that exhibits rotational motion. One exact solution that is everywhere regular in the exterior and interior regions of the rotating source is the metric associated to a thin, slowly rotating mass shell as described by Cohen [31]. In the limit of small angular momentum this metric corresponds to the asymptotic form of Kerr's metric tensor. The main motivation for considering this metric is the construction of a realistic source for the exterior region of the Kerr space-time, and therefore to match the latter region to a singularity-free space-time. For a shell of radius $r_{0}$ and total mass $m=2 \alpha$ as seen by an observer at infinity, the metric reads

$$
\begin{aligned}
d s^{2}= & -V^{2} d t^{2}+\psi^{4}\left[d r^{2}+r^{2} d \theta^{2}\right. \\
& \left.+r^{2} \sin ^{2} \theta(d \phi-\Omega d t)^{2}\right]
\end{aligned}
$$

where 


$$
\begin{gathered}
V=\frac{r_{0}-\alpha}{r_{0}+\alpha}, \\
\psi=\psi_{0}=1+\frac{\alpha}{r_{0}}, \quad \Omega=\Omega_{0}=\text { const, }
\end{gathered}
$$

for $r<r_{0}$, and

$$
\begin{gathered}
V=\frac{r-\alpha}{r+\alpha}, \\
\psi=1+\frac{\alpha}{r}, \quad \Omega=\left(\frac{r_{0} \psi_{0}^{2}}{r \psi^{2}}\right)^{3} \Omega_{0},
\end{gathered}
$$

for $r>r_{0}$.

The set of tetrad fields that satisfy conditions (4.4a),(4.4b) is given by

$$
e_{a \mu}=\left(\begin{array}{cccc}
-V & 0 & 0 & 0 \\
\Omega r \psi^{2} \sin \theta \sin \phi & \psi^{2} \sin \theta \cos \phi & r \psi^{2} \cos \theta \cos \phi & -r \psi^{2} \sin \theta \sin \phi \\
-\Omega r \psi^{2} \sin \theta \cos \phi & \psi^{2} \sin \theta \sin \phi & r \psi^{2} \cos \theta \sin \phi & r \psi^{2} \sin \theta \cos \phi \\
0 & \psi^{2} \cos \theta & -r \psi^{2} \sin \theta & 0
\end{array}\right)
$$

The determinant of $e_{a \mu}$ is $e=V r^{2} \psi^{6} \sin \theta$.

The nonvanishing components of the torsion tensor that are needed in the following read

$$
\begin{gathered}
T_{12}^{(1)}=r \partial_{r} \psi^{2} \cos \theta \cos \phi, \\
T_{12}^{(2)}=r \partial_{r} \psi^{2} \cos \theta \sin \phi, \\
T_{12}^{(3)}=-r \partial_{r} \psi^{2} \sin \theta, \\
T_{13}^{(1)}=-r \partial_{r} \psi^{2} \sin \theta \sin \phi, \\
T_{13}^{(2)}=r \partial_{r} \psi^{2} \sin \theta \cos \phi .
\end{gathered}
$$

The anti-symmetric components $\Pi^{[i k]}$ can be easily evaluated. We obtain

$$
\Pi^{[13]}(r, \theta, \phi)=4 k \alpha \frac{\Omega}{V} \psi \sin \theta,
$$

for $r>r_{0}, \quad \Pi^{[13]}(r, \theta, \phi)=0$ for $r<r_{0}$, and $\Pi^{[12]}(r, \theta, \phi)$ $=\Pi^{[23]}(r, \theta, \phi)=0$ for any value of $r$. In Cartesian coordinates the only nonvanishing component of the total angular momentum is given by

$$
\begin{aligned}
M^{12} & =2 \int d^{3} x \Pi^{[12]}(x, y, z) \\
& =4 \pi \int_{0}^{\pi} d \theta \int_{0}^{\infty} d r r \sin ^{2} \theta \Pi^{[13]}(r, \theta, \phi) \\
& =\alpha \int_{0}^{\pi} d \theta \sin ^{3} \theta \int_{r_{0}}^{\infty} d r r \psi \frac{\Omega}{V} .
\end{aligned}
$$

The integral above is finite, well behaved and can be exactly computed. However, we are interested only in the limit $r_{0} \gg \alpha$, in which case Cohen identifies $J=1 / 2\left(r_{0} \psi_{0}^{2}\right)^{3} \Omega_{0}$ as the Newtonian value for the angular momentum of a rotating mass shell [31]. In this limit the calculation is straightforward. We find

$$
M^{12} \simeq \frac{8 \alpha}{3 r_{0}} J=\frac{4 m}{3 r_{0}} J
$$

We identify $M^{12}$ as the angular momentum of the gravitational field. Substituting the expression of $J$ in Eq. (6.7) and considering that in the limit $r_{0} \gg \alpha$ we have $\psi_{0}=1$ $+\alpha / r_{0} \simeq 1$, we arrive at

$$
M^{12}=\left(\frac{2}{3} m r_{0}^{2}\right) \Omega_{0}
$$

$\Omega_{0}=\Omega\left(r_{0}\right)$ is the induced angular velocity of inertial frames inside the shell [32]. The term between the parentheses in the expression above corresponds to the moment of inertia of a rotating shell of radius $r_{0}$ and mass $m$. For small $\alpha, \Omega_{0}$ and the angular velocity of the shell $\omega_{s}$ are related via $\Omega_{0}$ $=\omega_{s}\left(4 m / 3 r_{0}\right)$ [32]. Therefore in the Newtonian limit $r_{0}$ $\gg \alpha$ we have $M^{12}=\left(\Omega_{0} / \omega_{s}\right) J$, where $J=(2 / 3) m r_{0}^{2} \omega_{s}$.

The metric tensor (6.4) is likely to be the only exact solution of Einstein's equations whose expression for the classical angular momentum of the source is precisely known.

In order to assess the significance of the above result, we will evaluate the angular momentum associated to the metric tensor (6.4) by means of Komar's integral $Q_{K}$ [29],

$$
Q_{K}=\frac{1}{8 \pi} \oint_{S} \sqrt{-g} \varepsilon_{\alpha \beta \mu \nu} \nabla^{[\alpha} \xi^{\beta]} d x^{\mu} \wedge d x^{\nu}
$$

where $S$ is a spherical surface of radius $R \rightarrow \infty, \quad \xi^{\mu}$ is the Killing vector field $\xi^{\mu}=\delta_{3}^{\mu}$ and $\nabla$ is the covariant derivative constructed out of the Christoffel symbols $\Gamma_{\mu \nu}^{\lambda}$. The integral $Q_{K}$ reduces to 


$$
Q_{K}=\frac{1}{2 \pi} \oint_{S} \sqrt{-g} g^{0 \mu} \Gamma_{\mu 3}^{1} d \theta d \phi
$$

By substituting Eq. (6.4) and taking the limit $S \rightarrow \infty$ we obtain

$$
Q_{K}=\frac{4}{3}\left(r_{0} \psi_{0}^{2}\right)^{3} \Omega_{0} \simeq \frac{4}{3} r_{0}^{3} \Omega_{0}=\frac{16}{9} m r_{0}^{2} \omega_{s}=\frac{8}{3} \mathrm{~J} .
$$

In the equation above we are considering $r_{0} \gg \alpha$. We observe that definitions (6.3) and (6.9) yield distinct results. In order to make clear the distinction it is useful to rewrite both espressions (6.7) and (6.11) in laboratory (CGS) units. Thus we make $m=\left(G / c^{2}\right) M$ and $\omega_{s}=\Omega_{s} / c$, where $M$ is given in grams, and $\Omega_{s}$ in radians per second. In addition, we make the replacement $1 /(16 \pi) \rightarrow c^{3} /(16 \pi G)$ in the multiplicative factor of both expressions, in order to yield the correct dimension to the integrals. We arrive at

$$
\begin{gathered}
M^{12}=\left(\frac{G}{c^{2}}\right) \frac{4 M}{3 r_{0}}\left(\frac{2}{3} M r_{0}^{2} \Omega_{s}\right), \\
Q_{K}=\frac{8}{3}\left(\frac{2}{3} M r_{0}^{2} \Omega_{s}\right) .
\end{gathered}
$$

We note that $G / c^{2}=0,74 \times 10^{-28} \mathrm{~g} / \mathrm{cm}$. Both expressions have angular momentum units.

One expects the gravitational angular momentum to be of the order of magnitude of the intensity of the gravitational field. We observe that Komar's integral yields a value proportional to the angular momentum of the source, whereas $M^{12}$ is much smaller than $Q_{K}$. Indeed, the gravitational field of a mass shell of typical laboratory values is negligible, and consequently the gravitational angular momentum should be negligible as well. Therefore $M^{12}$ yields a realistic value for the angular momentum of the gravitational field, in contrast to $Q_{K}$.

The advantage of definition (6.4) is that it does not depend on the existence of Killing vector fields. The conclusion is that the angular momentum of the space-time of a rotating mass shell, according to the definition (6.3), is proportional to the induced angular velocity $\Omega_{0}$ of inertial frames.

The investigations carried out so far in the context of the Kerr solution are not yet conclusive. Although the calculations in the Boyer-Lindquist coordinates are extremely intricate, the indications are that $M^{12}$ diverges. Considering the metric tensor given by Eq. (4.5) and the related definitions, we calculate the antisymmetric components of the momenta $\Pi^{[i k]}$ in the time gauge, i.e., out of tetrads (4.9). They are given by

$$
\begin{gathered}
\Pi^{[12]}(r, \theta, \phi)=0, \\
\Pi^{[13]}(r, \theta, \phi)=\frac{k \chi \sin \theta}{\sqrt{\psi^{2} \Sigma^{2}+\chi^{2} \sin ^{2} \theta}} \\
\\
\times\left(1+\frac{\rho^{2}}{\Sigma}-\frac{\sqrt{\Delta}}{\Sigma} \partial_{r} \Sigma\right),
\end{gathered}
$$

$$
\begin{aligned}
\Pi^{[23]}(r, \theta, \phi)= & \frac{k \chi}{\sqrt{\Delta\left(\psi^{2} \Sigma^{2}+\chi^{2} \sin ^{2} \theta\right)}} \\
& \times\left[\cos \theta\left(\frac{\rho^{2}}{\Sigma}-1\right)-\frac{\sin \theta}{\Sigma} \partial_{\theta} \Sigma\right] .
\end{aligned}
$$

Transforming to Cartesian coordinates we obtain

$$
\begin{aligned}
M^{12}= & \int d^{3} x \Pi^{[12]}(x, y, z) \\
= & 2 \pi \int_{0}^{\infty} d r \int_{0}^{\pi} d \theta\left[r \sin \theta \Pi^{[13]}(r, \theta, \phi)\right. \\
& \left.+r^{2} \sin \theta \cos \theta \Pi^{[23]}(r, \theta, \phi)\right],
\end{aligned}
$$

and $M^{13}=M^{23}=0$. The evaluation of Eq. (6.13) out of expressions (6.12) yields a divergent result. The latter is positively and negatively divergent in the external $\left(r_{+}\right)$and internal $\left(r_{-}\right)$horizons of the black hole, respectively. Moreover, in the region $r_{-}<r<r_{+}, M^{12}$ acquires an imaginary component. A possible interpretation is that the Boyer-Lindquist coordinates are not suitable to the present analysis. In any way, integration over the whole spacelike section of the Kerr space-time is a nontrivial operation.

It must be noted that the Kerr black hole has no classical analog. The interpretation of the angular momentum parameter $a$ of the Kerr solution is not straightforward, since in the Newtonian theory of gravitation the gravitational field of a body does not depend on its rotational motion. The parameter $a$ is identified with the angular momentum per unit mass of the source only after reducing the exterior region of the Kerr metric to a Lense-Thirring type metric by successive approximations [33].

\section{DISCUSSION}

In this paper we have investigated the definitions of energy and angular momentum of the gravitational field that arise in the Hamiltonian formulation of the TEGR. We have compared the most important achievement, i.e., the calculation of the irreducible mass of the Kerr black hole, with the result previously obtained in the framework of the same theory, but with the Hamiltonian formulation established under the a priori imposition of the time gauge condition. The two results agreed. In fact, both energy expressions coincide by requiring the time gauge condition, if the latter is imposed a posteriori in the $a=(0)$ component of expression (3.3).

The relevance of Eq. (5.4) is further enhanced if we observe that the Brown-York method [3] for the evaluation of quasilocal gravitational energy fails in obtaining a value close to the irreducible mass of the Kerr black hole. Although the calculations in the framework of this method are quite intricate, recently it has been carried out [34]. It has been shown that the gravitational energy within $r_{+}$is close to $2 M_{i r r}$ only for $a / m<0.5$ (Fig. 1 of Ref. [34]).

Definitions for the gravitational energy in the context of the teleparallel equivalent of general relativity have already 
been proposed in the literature. In Ref. [11] an expression for the gravitational energy arises from the surface term of the total Hamiltonian [Eqs. (3.18) and (3.19) of Ref. [11]]. A similar quantity is suggested in Ref. [35], according to Eq. (3.8) of the latter reference. Both expressions are equivalent to the integral form of the total divergence of the Hamiltonian density developed in Ref. [18] [Eq. (27) of the latter reference],

$$
E=\int_{V \rightarrow \infty} d^{3} x \partial_{k}\left(e_{a 0} \Pi^{a k}\right)=\oint_{S \rightarrow \infty} d S_{k}\left(e_{a 0} \Pi^{a k}\right) .
$$

The three expressions yield the same value for the total energy of the gravitational field. However, since these three expressions contain the lapse function in the integrand, none of them is suitable to the calculation of the irreducible mass of the Kerr black hole, in which case we consider a finite surface of integration, because the lapse funtion vanishes on the external event horizon of the black hole (recalling the $3+1$ decomposition in Sec. III, $e^{a}{ }_{0}=\eta^{a} N+{ }^{3} e^{a}{ }_{i} N^{i}$. In the time gauge we have $\eta^{a}=\delta_{(0)}^{a}$ and $\left.e^{(0)}{ }_{i}=0\right)$. The energy expressions of Refs. $[11,35]$ are not to be applied to a finite surface of integration; rather, they yield the total energy of the space-time.

The energy expression (3.3) is defined with respect to a given reference space. Tetrad fields that satisfy conditions (4.4a),(4.4b) establish a unique reference space-time that is neither boost related nor rotating with respect to the physical space-time. These conditions uniquely associate a set of tetrad fields to an arbitrary metric tensor. Therefore in the present framework it does not suffice to assert that the reference space-time is Minkowski's space-time. It is also necessary to enforce the soldering of the reference space-time to the physical space-time by means of Eqs. (4.4a),(4.4b). We conjecture that for a given space volume the latter conditions yield the minimum value for the energy expression (3.3).

\section{ACKNOWLEDGMENTS}

T.M.L.T. and K.H.C.B. are grateful to the Brazilian agency CAPES, and J.F.R.N. to FAPESP, for financial support.

\section{APPENDIX}

We present here the components of the torsion tensor obtained out of the tetrad configuration Eq. (4.7), which satisfies Møller's weak field approximation:

$$
\begin{aligned}
& T_{01}^{(0)}=\sqrt{1+M^{2} y^{2}}\left(\frac{\psi}{\rho^{2}} \partial_{r} \rho-\frac{1}{\rho} \partial_{r} \psi\right) \\
& -\frac{\psi M y}{\rho \sqrt{1+M^{2} y^{2}}}\left(y \partial_{r} M+M \partial_{r} y\right) \\
& T_{13}^{(0)}=\frac{y N \chi}{\rho \psi} \sin ^{2} \theta\left(\frac{1}{y} \partial_{r} y+\frac{1}{\chi} \partial_{r} \chi+\frac{1}{N} \partial_{r} N-\frac{1}{\rho} \partial_{r} \rho-\frac{1}{\psi} \partial_{r} \psi\right) \text {, } \\
& T_{01}^{(1)}=-\frac{y \chi}{\rho \Sigma} \sin \theta \sin \phi\left(\frac{1}{\chi} \partial_{r} \chi+\frac{1}{y} \partial_{r} y-\frac{1}{\rho} \partial_{r} \rho-\frac{1}{\Sigma} \partial_{r} \Sigma\right), \\
& T_{03}^{(1)}=-\frac{y \chi}{\rho \Sigma} \sin \theta \cos \phi, \\
& T_{12}^{(1)}=\cos \theta \cos \phi\left(\partial_{r} \rho-\frac{\rho}{\sqrt{\Delta}}\right)-\frac{1}{\sqrt{\Delta}} \sin \theta \cos \phi \partial_{\theta} \rho, \\
& T_{13}^{(1)}=\sin \theta \sin \phi\left[\frac{\rho}{\sqrt{\Delta}}-\frac{\Sigma}{\rho} \sqrt{1+N^{2} M^{2} y^{2}}\left(\frac{1}{\Sigma} \partial_{r} \Sigma-\frac{1}{\rho} \partial_{r} \rho\right)\right. \\
& \left.-\frac{\sum N^{2} M^{2} y^{2}}{\rho \sqrt{1+N^{2} M^{2} y^{2}}}\left(\frac{1}{N} \partial_{r} N+\frac{1}{M} \partial_{r} M+\frac{1}{y} \partial_{r} y\right)\right] \\
& T_{01}^{(2)}=\frac{y \chi}{\rho \Sigma} \sin \theta \cos \phi\left(\frac{1}{\chi} \partial_{r} \chi+\frac{1}{y} \partial_{r} y-\frac{1}{\rho} \partial_{r} \rho-\frac{1}{\Sigma} \partial_{r} \Sigma\right) \text {, } \\
& T_{03}^{(2)}=-\frac{y \chi}{\rho \Sigma} \sin \theta \sin \phi \text {, } \\
& T_{12}^{(2)}=\cos \theta \sin \phi\left(\partial_{r} \rho-\frac{\rho}{\sqrt{\Delta}}\right)-\frac{1}{\sqrt{\Delta}} \sin \theta \sin \phi \partial_{\theta} \rho, \\
& T_{13}^{(2)}=-\sin \theta \cos \phi\left[\frac{\rho}{\sqrt{\Delta}}-\frac{\Sigma}{\rho} \sqrt{1+N^{2} M^{2} y^{2}}\left(\frac{1}{\Sigma} \partial_{r} \Sigma\right.\right. \\
& \left.-\frac{1}{\rho} \partial_{r} \rho\right)-\frac{\Sigma}{\rho \sqrt{1+N^{2} M^{2} y^{2}}}\left(\frac{1}{N} \partial_{r} N+\frac{1}{M} \partial_{r} M\right. \\
& \left.\left.+\frac{1}{y} \partial_{r} y\right)\right] \\
& T_{12}^{(3)}=-\sin \theta\left(\partial_{r} \rho-\frac{\rho}{\sqrt{\Delta}}\right)-\frac{1}{\sqrt{\Delta}} \cos \theta \partial_{\theta} \rho .
\end{aligned}
$$

[1] C. Møller, Tetrad Fields and Conservation Laws in General Relativity, Proceedings of the International School of Physics "Enrico Fermi," edited by C. Møller (Academic Press, London, 1962); Conservation Laws in the Tetrad Theory of Gravitation, Proceedings of the Conference on Theory of Gravitation, Warszawa and Jablonna, 1962, NORDITA Publications No. 136 (Gauthier-Villars, Paris, and PWN-Polish Scientific Publishers, Warszawa, 1964).
[2] S. V. Babak and L. P. Grishchuk, Phys. Rev. D 61, 024038 (2000).

[3] J. D. Brown and J. W. York, Jr., Phys. Rev. D 47, 1407 (1993); S. A. Hayward, ibid. 49, 831 (1994); 53, 1938 (1996); J. D. Brown, S. R. Lau, and J. W. York, Jr., ibid. 59, 064028 (1999); C.-C. Chang, J. M. Nester, and C. M. Chen, Phys. Rev. Lett. 83, 1897 (1999).

[4] R. Weitzenböck, Invarianten Theorie (Nordhoff, Groningen, 
1923).

[5] J. A. Schouten, Ricci Calculus, 2nd ed. (Springer-Verlag, London, 1954), p. 142.

[6] A. Einstein, Riemannsche Geometrie unter Aufrechterhaltung des Begriffes des Fernparallelismus (Riemannian Geometry with Maintaining the Notion of Distant Parallelism), Sitzungsberichte der Preussischen Akademie der Wissenshcaften, Phys.-Math. Klasse, 1928, pp. 217-221; Auf die RiemannMetrik und den Fernparallelismus gegründete einheitliche Feldtheorie (Unified Field Theory based on Riemann Metrics and Distant Parallelism), Mathematische Annalen 102, 685 (1930) (English translations available under www.lrz.de/ aunzicker/ae1930.html).

[7] K. Hayashi, Phys. Lett. 69B, 441 (1977); K. Hayashi and T. Shirafuji, Phys. Rev. D 19, 3524 (1979); 24, 3312 (1981).

[8] F. W. Hehl, in Proceedings of the 6th School of Cosmology and Gravitation on Spin, Torsion, Rotation and Supergravity, Erice, 1979, edited by P. G. Bergmann and V. de Sabbata (Plenum, New York, 1980); F. W. Hehl, J. D. McCrea, E. W. Mielke, and Y. Ne'eman, Phys. Rep. 258, 1 (1995).

[9] W. Kopczyński, J. Phys. A 15, 493 (1982); Ann. Phys. (N.Y.) 203, 308 (1990).

[10] F. Müller-Hoissen and J. Nitsch, Phys. Rev. D 28, 718 (1983); Gen. Relativ. Gravit. 17, 747 (1985).

[11] J. M. Nester, Int. J. Mod. Phys. A 4, 1755 (1989).

[12] J. M. Nester, J. Math. Phys. 33, 910 (1992).

[13] J. W. Maluf, J. Math. Phys. 35, 335 (1994).

[14] V. C. de Andrade and J. G. Pereira, Phys. Rev. D 56, 4689 (1997).

[15] J. Schwinger, Phys. Rev. 130, 1253 (1963).

[16] J. W. Maluf, J. Math. Phys. 36, 4242 (1995).

[17] V. C. de Andrade, L. C. T. Guillen, and J. G. Pereira, Phys. Rev. Lett. 84, 4533 (2000).

[18] J. W. Maluf and J. F. da Rocha-Neto, Phys. Rev. D 64, 084014 (2001).
[19] R. Arnowitt, S. Deser, and C. W. Misner, in Gravitation: An Introduction to Current Research, edited by L. Witten (Wiley, New York, 1962).

[20] R. P. Kerr, Phys. Rev. Lett. 11, 237 (1963).

[21] D. Christodoulou, Phys. Rev. Lett. 25, 1596 (1970).

[22] G. Bergqvist, Class. Quantum Grav. 9, 1753 (1992).

[23] J. W. Maluf, E. F. Martins, and A. Kneip, J. Math. Phys. 37, 6302 (1996).

[24] J. W. Maluf and J. F. da Rocha-Neto, J. Math. Phys. 40, 1490 (1999).

[25] J. A. Schouten, Tensor Analysis for Physicists, 2nd ed. (Dover, New York, 1989), p. 82.

[26] R. H. Boyer and R. W. Lindquist, J. Math. Phys. 8, 265 (1967).

[27] L. D. Landau and E. M. Lifshitz, The Classical Theory of Fields (Pergamon, Oxford, 1980). We note that the first Russian edition dates from 1939.

[28] P. G. Bergmann and R. Thomson, Phys. Rev. 89, 401 (1953).

[29] A. Komar, Phys. Rev. 113, 934 (1959); J. Winicour, "Angular Momentum in General Relativity," in General Relativity and Gravitation, edited by A. Held (Plenum, New York, 1980); A. Ashtekar, "Angular Momentum of Isolated Systems in General Relativity," in Cosmology and Gravitation, edited by P. G. Bergmann and V. de Sabbata (Plenum, New York, 1980).

[30] K. Hayashi and T. Shirafuji, Prog. Theor. Phys. 73, 54 (1985); M. Blagojević and M. Vasilić, Class. Quantum Grav. 5, 1241 (1988); T. Kawai, Phys. Rev. D 62, 104014 (2000); T. Kawai, K. Shibata, and I. Tanaka, Prog. Theor. Phys. 104, 505 (2000).

[31] J. M. Cohen, J. Math. Phys. 8, 1477 (1967).

[32] D. R. Brill and J. M. Cohen, Phys. Rev. 143, 1011 (1966).

[33] R. Adler, M. Bazin, and M. Schiffer, Introduction to General Relativity (McGraw-Hill, Tokyo, 1975), p. 257.

[34] M. H. Dehghani and R. B. Mann, Phys. Rev. D 64, 044003 (2001).

[35] M. Blagojević and M. Vasilić, Phys. Rev. D 64, 044010 (2001). 